\title{
What can GIS do?
}

Forthcoming in ACME: An International Journal for Critical Geographies

Nick Lally

Department of Geography

University of Kentucky

nicklally@uky.edu

\begin{abstract}
This special issue is organized around the deceptively simple question: "what should the doing of critical GIS look like?" Instead of declaring what path critical GIS should take, I instead argue that we do not know what GIS can do. I suggest that we hold a place for doubt and unknowing, acknowledging that the potentials and possibilities of GIS do not preexist practice, but rather, critical GIS emerges in the doing and practice of GIS. Following this argument, I make three main claims in the article. First, I argue that understanding the possibilities for GIS requires being attentive to how particular instantiations of GIS connect with social and material relations. Second, I argue that the doing of critical GIS might use these existing limitations as a starting point for the remaking of GIS. Third, I argue that the doing of critical GIS has an important role to play in understanding how GIS is used within infrastructures of governance. I conclude by suggesting that the doing of critical GIS takes meaningful form through experimentation, openness to the encounter, and linking with existing situated practices and theories.
\end{abstract}

Keywords: GIS, geographical imagination systems, community geographies, speculative computing, algorithmic governance 


\section{Introduction}

This special issue and the workshop it arose from were organized around a prompt that asked: "what should the doing of critical GIS look like?" This is a deceptively hard question to answer, not just because of the multitude of exciting and generative practices that have developed under the rubric of critical GIS. And not just because I hesitate to make such normative statements in turbulent times that require an openness to new and imaginative approaches to social and spatial problems. This question is also difficult to answer because I do not think we even know what GIS can do.

Claiming that we do not know what GIS can do is not intended to be a flippant remark. Rather, in recognizing the unknown and the possible, I hope to suggest other paths for critical GIS practices. Attending every effort to chart a course for critical GIS, I suggest that we hold a place for doubt and unknowing, acknowledging that the potentials and possibilities of GIS do not preexist practice. As such, I argue that critical GIS emerges in the doings and practices of GIS. But this doing-critique might break with previous modes and understandings of existing GIS technologies. For us to really begin to understand what GIS can make possible, I think we need expansive, experimental, and exploratory approaches to scholarship. In other words, recognizing that we do not know what GIS can do might lead us to change and enroll GIS in ways more in accord with the diverse theoretical and political commitments of human geographers, activists, and others committed to social change.

In the following sections, I highlight specific examples of scholars and activists confronting the limitations of GIS. I argue that these limitations might lead us to reimagine what is technically and socially possible within these socio-technical collaborations. While such projects are necessarily context dependent and site specific, some general examples can be illustrative of the gap between GIS and particular theoretical orientations of geography. We can imagine, for example, a community geography project whose participants hold complex notions of relationality that enroll humans and non-humans, near and far. When confronted with a GIS, these notions of relationality might need to be reduced to discrete datasets that can be overlaid and understood as proximate only in simple Euclidean measures of distance. Similarly, a project grounded in expansive and relational understandings of ontology might confront spatial technologies that insist on the individuated and precisely locatable points of GIS. In both cases, there is gap between the grounding knowledges of a project and the abilities of GIS to incorporate those ways of knowing. The translations across these gaps are key to understanding what GIS can do, both in terms of how GIS is differentially actualized and in what potentials it might contain.

To further elucidate what I mean when I say that we don't know what GIS can do, I make three main claims. First, I argue that understanding the possibilities for GIS requires being attentive to how particular instantiations of GIS connect with social and material relations. Studying the often fraught translations that arise in community mapping projects, for example, can lead to broader understandings of existing limitations and future possibilities for GIS. Second, I argue that the doing of critical GIS might use these existing limitations as a starting point for the remaking of GIS. Using my own experiment with remaking GIS technologies as an example, I suggest how GIS might be transformed to engage more deeply with space as a theoretical matter of concern. Third, I argue that the doing of critical GIS has an important role to play in understanding how GIS is used within infrastructures of governance. Considering the lack of public access to the methods and algorithms deployed in this work, I suggest an exploratory approach that attempts to engage with these systems 
using researchers' knowledge of GIS. I conclude by suggesting that the doing of critical GIS takes meaningful form through experimentation, openness to the encounter, and linking with existing situated practices and theories.

\section{Doing GIS}

While GIS is a collection of apparatuses that enable certain types of epistemological orientations, they always builds upon existing knowledges, epistemologies, practices, and materialities that they connect with. For example, scholars using GIS in community-oriented projects have shown how existing organizational contexts, group cultures, and individual situated knowledges shape how GIS is adopted and understood (Sieber 2004; Elwood 2006; Elwood and Ghose 2001) and how that can result in very different understandings of the same phenomena. Robert Norheim (2001) shows how acreage estimates of old growth forests by the US Forest Service and a conservationist group differed significantly, which the author traces back to institutional differences. Similarly, work in Science and Technology Studies (STS) has insisted on the necessity of understanding the social and material relations that connect with a technology to give it the power to produce knowledge. STS offers methods of studying technologies within the context of their use, which often diverges from what was intended by their designers (Suchman et al. 1999; Poore 2003).

GIS contributes to knowledge of the world within shifting networks of practice, which might differ from or exceed the intentions or expectations of those engaged with more formalized avenues of GIS research. In other words, we can't foresee every possibility for GIS until it connects with the social and material relations that make it matter (and vice versa). These relations, it should be noted, are constantly in flux, so mapping technologies unfold and make meaning within shifting networks that continuously modulate relations of knowledge production (Kitchin, Gleeson, and Dodge 2013). Understanding how GIS is put into practice and often detourned for the needs of a project can lead to an expanded understanding of what a GIS can do.

Thus, to understand what GIS can do requires an openness to the encounter-an openness that recognizes that existing struggles and practices will shape GIS as much as GIS might contribute to those struggles. In other words, I am suggesting that spaces that bring together community groups, activists, academics, and/or others around GIS (like the one that inspired this special issue) are great spaces for experiments with GIS as a form and a practice, provided that participants are ready to listen and adapt to other ways of knowing.

This intervention, I must note, is largely directed at academics like myself, who might enter a project with preconceived notions of the best methods for approaching a particular problem. In reflecting on the Detroit Geographical Expeditions, Gwendolyn Warren (1971) writes of meeting with the academic geographers of Wayne State University: "Out of all the stuff that they were saying to us, we wondered how we could take a little bit out of all that 'bull' and make it useful" (10). She continues,

Everybody was talking in terms of how everything was bad; the garbage was not picked up; the place looked bad and things of that nature. I got mad. All of these students failed to see one of the deadliest things. I saw there were no candy wrappers, that the children in that area were starved for candy and that they were hungry and that when the popsicle man came down the street all the children looked out the windows but nobody went for the truck. (10) 
The "bull" of academic geographic theories combined with preconceived notions of what constituted community concerns rubbed activists the wrong way as academics failed to understand what was really important to know about this place. And while I am not suggesting academics abandon their expertise in these situations (because as Warren observed, "we took your science and we are using your science in our everyday fight to survive in the City" [Warren 1971, 10]), I am suggesting holding a place for doubt, recognizing that existing epistemologies might conflict with those offered by eager GISers. Recognizing this epistemological gap highlights the negotiations that are necessary in working across various types of difference. These negotiations around knowledge are not easy and take time that might conflict with the timeframes of academia, but are one of a host of challenges in building trust in community-academic partnerships (Robinson, Block, and Rees 2017).

Warren's quote castigating students makes me think of my own experience teaching GIS and the tendency of so many students to center various types of deprivation and dispossession in understanding a space, usually by comparing various datasets produced by the US Census. Following Warren, this dynamic is an easy setup for failure in academic-community projects. Katherine McKittrick (2011) similarly warns of the tendency for academics to only center violence, deprivation, and loss in the study of Black life. Instead, as McKittrick argues, what if human relationality were at the center of such projects instead of binaries of possession/dispossession or with/without? What this might look like, however, is hard to prefigure in isolation of particular communities, whether in an academic GIS lab or the delivery of predetermined methods.

In community mapping projects, the challenge of fitting existing knowledge into a GIS framework often becomes an intractable problem. Michael Brown and Larry Knopp (2008), for example, describe the "epistemological tensions" that arose in mapping a queer history of Seattle in an action-research project. They observe that participants "had different and changing epistemological approaches to cartography" and "each of us 'knew' the data differently (or even multiply)" (49). In fitting data to GIS, the authors document challenges of categorization, but also in representing "connectivity, fluidity, multiplicity, and multiple scales" (50)—concepts that are difficult or impossible to translate into common GIS operations. Similarly, Robert Rundstrom (1995) observes the inability for GIS to incorporate Indigenous knowledges that value relationality, ambiguity, and anomalous data that resist categorization. GIS under these conditions, according to Rundstrom, becomes a "tool for epistemological assimilation" (45). What if these epistemological clashes so common to community mapping projects became the starting point for reimagining what a GIS can do?

While the above encounters focus on academic GIS practitioners working with community partners, various moments of translation between phenomena and the data operations of GIS might yield similarly productive epistemological gaps. The collection, processing, sorting, manipulation, representation, and interpretation of data all encompass complex encounters between human and non-human participants who are enrolled within the apparatuses of GIS. At every moment of encounter, an openness to rethinking the foundations of GIS might lead to other ways that human and non-human participants might be induced to speak through, with, and across various technologies.

In these fraught translations between community knowledges, phenomena, and data enrolled within a particular GIS, we might imagine other possibilities for the technologies of GIS. As Renee Sieber (2004) argues: "we need a technology that can better accommodate a world of disagreement, passion, complexity, and redundancy" (29). Sieber and others have argued for remaking the technologies of GIS to better incorporate social, political, cultural, and theoretical commitments that 
are seemingly incommensurate with GIS as it exists (Sieber 2004; Gahegan 2018; Gieseking 2018). In summary, my first suggestion for the doing of critical GIS is to understand how GIS is put into use within different contexts, with special attention to the "epistemological tensions" that arise and reveal the limitations of existing GIS. These moments of tension can then inform the remaking of GIS, which is my second suggestion for the doing of critical GIS.

\section{From GIS to gis}

In response to the limited conceptual framework of GIS, Luke Bergmann and I have suggested a shift from GIS as we know it to the expanded field of geographical imagination systems (gis) (Bergmann and Lally 2021). gis, in our conceptualization, is not a negation of GIS, but rather, an expansion of existing GIS in order to incorporate other ways of knowing, interpreting, and playing with space. In this conceptualization, we build upon a rich history of thought that similarly looks beyond the limits of existing GIS in order to imagine other possibilities. Our own prototype gis is informed by the gap between spatial theory in human geography and the inability to realize many of those ideas in available GIS software (Lally and Bergmann 2021).

We are inspired by conceptualizations of space in human geography, often theorized as being co-produced through relations between various phenomena. Yet commonly available mapping GIS treat space as an empty container to be filled with discrete, locatable objects. This Euclidean description of mathematical space is largely irreconcilable with the rich body of spatial theory that has developed within and beyond human geography. To bridge these practices, we have developed a prototype called enfolding (see: https://github.com/FoldingSpace/enfolding), which can visualize relational, topological, and other non-Euclidean spaces. Our goal for gis is to build accessible tools that can facilitate creative visualizations of space for scholars in human geography, digital humanities, and other disciplines who want to use maps in their research without being limited by Cartesian coordinate systems (Lally and Bergmann 2021). In enfolding, users can redefine Cartesian measures of distance, which the software will use to bend and fold space using multidimensional scaling in two or three dimensions. Additionally, users may choose to put two maps in relation, creating a wormhole between two spaces that may be distant in Euclidean understandings of distance. Both of these operations, and others available in the software, allow for creative visualizations of space that exceed what is possible in most GIS software.

There have been a number of one-off maps that do similar work and from which we draw inspiration, but they generally take specialized skills and tools. For us, an important part of this project is allowing a user to play with spatial forms using accessible tools. In that way, they are playing and experimenting with spatial theory, which can hopefully be a generative moment that shifts how space is thought. It is also a way to play with gis and note the limits of GIS at the edges of visualization and representation, and the sometimes fraught relationships between representation and theory.

My background in visual arts informs my approach to visual methods. The tension between representation and theory is central to much of my thinking and is, I believe, worth pushing to its limits, often with the intention of exploding those limits. During many key moments in art history, art has similarly been invested in its own overcoming, always asking itself: what else can art do or be (Holmes 2009)? gis is similarly a challenge for GIS to break its own assumptions and expand our notions of what a GIS can do. While scientific refinement has been central to the development of GIS methods and theories, here I am suggesting an experimental and interpretive approach to 
developing new tools and ways of thinking through gis. In other words, experiments to expand the possibilities of GIS might rely more on the expressive possibilities and creative potentials of the medium than the refinement of scientific or statistical methods. This suggestion is informed and influenced by experiments in speculative computing and design. For example, Tara McPherson's (2018) Vectors Lab centers aesthetic and socio-theoretical questions in producing experimental software that attempts to break from rigid, modular structures of computing. And Johanna Drucker's (2009) work in speculative computing-which insists on interpretive, aesthetic, and humanistic approaches to producing new paradigms for software-has been generative in my collaboration with Bergmann.

While my own engagements with the creation of gis technologies is informed by academic spatial theory and speculative computing, I recognize that this is necessarily a narrow horizon of possibility for remaking GIS. This is why I argue for the importance of being attentive to how GIS is actually put into use and the "epistemological tensions" that arise in the previous section. These moments of confusion, frustration, translation, and détournement can be the starting points for imagining other possibilities for gis.

While the software Bergmann and I produce has resonances with some specific ways of understanding space, the larger project of gis should rely on a plurality of spatial epistemologies. Different ways of understanding space would likely benefit from a diverse set of expressions in gis. We might imagine, for example, a plurality of ways to visualize space variously conceptualized as situated, relational, unfinished, or dynamic. How can the doing of critical GIS, and particularly the doings that audiences of academic texts might engage, work to remake GIS as a means to support other ways of knowing? As I suggest here, some of these other possibilities might emerge in collaboration with community projects that run into the limits of GIS. What expanded modes of participatory research might emerge if those limits were only beginnings? What if limits were subject to not only the reflexivity of researchers, but also the possibility of their overcoming? Existing limits have often inspired new horizons of possibility for GIS — in this paper, I am suggesting that the doing of critical GIS produce the context and conditions that may contribute to the expansion of GIS in ways that increasingly support the work of those committed to humanistic inquiry and social change.

If mappings always unfold within particular, shifting contexts (Kitchin, Gleeson, and Dodge 2013), shifting those contexts can expand the possibilities for gis to unfold. In other words, new possibilities for gis can develop through the doing of critical GIS-here conceptualized through community and related geography projects found in academic literature-which inform the remaking of existing technologies. This remaking will likely require new alliances between those engaged in such projects and those engaged with software development who may be brought together in exploratory collaborations.

Within the context of these collaborations, gis can be productively thought of as a humble iteration of GIS, focused on listening, improvisation, and adaptation. gis might follow Anna Secor and Jess Linz's (2017) suggestion for becoming-minor: "Begin to play without knowing what will be played, how it will unfold, where it will end or start up again. Become absorbed. Detach from conventions of judgment, aesthetic and moral" (572). If GIS is a set of techniques backed by scientific methodologies, gis opens into more interpretive, humanistic way of knowing. A lowercase gis might tend to defer to community concerns and supplement rather than supplant other epistemological orientations. gis might then be thought of as existing within emerging feedback loops, as it is remade through community and scholarly engagement, informing other forms of theory and praxis, which then go onto informing future iterations of gis. 


\section{The secret lives of GIS}

While the iterative possibilities for a humanistic and socially-engaged gis is one vision for the doing of critical GIS, the fact that GIS (with an emphatic capital 'G') is increasingly enrolled within infrastructures of governance offers another urgent avenue of research for the doing of critical GIS. Ongoing critiques of the power of GIS have been central to the development of critical GIS (Schuurman 2000) and continue to inspire important critiques of the oppressive implementations of GIS. Brian Jordan Jefferson (2017b), for example, shows how Chicago's online mapping software encourages the decontextualization of crime away from structural considerations while simultaneously enrolling citizens within the apparatus of police surveillance. Additionally, Jefferson (2017a) argues that crime mapping provides scientific cover for racist police practices as it "legitimize both the data sets and the police practices that produce them" (3). Tracking the evolution of GIS as a tool for governance is certainly important, especially considering that for many, 'the call is coming from inside the house.' The adjacency of many critical geographers to the GIS technologies, discourses, and methods that become enrolled within infrastructure of governance might provide a unique perspective from which to study those infrastructures.

GIS used within systems of governance pose unique challenges for research. For example, there is often little public access to the methods and algorithms used within these systems. And even if there is, machine learning, big data, and the linking of disparate systems all tend to resist human comprehension (Amoore 2018). Consider, for example, predictive policing, where proprietary machine learning algorithms built into GIS systems and working on large datasets redistribute the geographical allocation of police patrols. Not only are many of these systems intentionally hidden from scrutiny, but how they are put into use is also largely hidden by the secretive nature of policing. Considering the systemic racism ubiquitous to policing in the United States and elsewhere, how GIS reflects, reifies, or otherwise becomes enrolled within racist systems of policing becomes an urgent question.

How do we then engage in critical GIS practices that confront and study practices like predictive policing, which are hidden, complex, and constantly shifting in time and space? We are necessarily always chasing new research that is reshaping what GIS can do, mapping out traces of data, and speculating about the effects and possibilities of such technologies. But there are always limits to understanding what GIS can do, especially when they become part of the militaryindustrial-governmental complex and intentionally hidden away from view. Despite these challenges, I think it's a worthwhile goal for critical GIS to poke at the edges of these systems, constantly asking what they are doing and producing. This might be a speculative approach informed by our own knowledge of and engagement with GIS.

Kristian Lum and William Isaac (2016), for example, created their own implementation of the algorithm used in the predictive policing software package PredPol ${ }^{1}$ and used it to analyze drug crimes in Oakland, California. The authors found that city's Black residents would be targeted by policing at twice the rate of white residents, reinforcing existing racial biases in policing. While critics are correct in pointing out that this type of data is not currently used in known predictive policing implementations, these sorts of experiments might be useful in preempting the misuse of software. By showing what is possible and drawing analogies with what might exist, the doing of critical GIS can be revealing of the growing complicity of GIS with oppressive forms of governance. In the

\footnotetext{
${ }^{1}$ The PredPol algorithm is described in an academic paper, which the authors were able to use in their study. Most innovative work in GIS research is never implemented into GIS software Gahegan (2018) making their implementation require specialized programming and statistical knowledge.
} 
context of emerging social movements, growing media scrutiny, and academic research, Santa Cruz, California — an early adopter of predictive policing software-banned the technology in the city (Guariglia 2020). While the reasons for banning this software are undoubtedly complex, critical analyses of the technology and its potential for racial bias certainly played a role.

One possible path for the doing of critical GIS is to continue to support the work of growing social movements (like the Stop LAPD Spying Coalition: https://stoplapdspying.org/) working to counter oppressive uses of mapping technologies. This suggestion, of course, is only one part of understanding what GIS can do. As I argue above, how these systems are actually put into practice is always fraught and contingent.

\section{Conclusion}

In this article, I have outlined how not knowing what GIS can do might open up new ways of conceptualizing the doing of critical GIS. In the first section, I highlighted how doing GIS in community contexts is often a fraught and contested practice that requires an openness to negotiating these tensions. In the second section, I argued that attention to tensions that arise can be productive in reimagining what GIS can do. I framed this reimagining of GIS as a shift to geographical imagination systems that embody an expanded conceptual grammar for knowing space. And finally, in the third section, I argued for the importance of studying how GIS is currently being used within various forms of governance and control. From this final research path, as Louise Amoore (2019) writes, we might "experiment, like the algorithmic models do, iteratively and recursively giving doubtful accounts of the output of a calculation" (163). While qualitatively different than the reimagining of GIS, this path similarly suggests an open, exploratory, and experimental engagement with GIS as a technology that engages it within particular instantiations, embedded within situated practices. 


\section{References}

Amoore, Louise. 2018. "Cloud Geographies: Computing, Data, Sovereignty.” Progress in Human Geography 42 (1): 4-24. doi:10.1177/0309132516662147.

- 2019. "Doubt and the Algorithm: On the Partial Accounts of Machine Learning." Theory, Culture \& Society 36 (6): 147-69. doi:10.1177/0263276419851846.

Bergmann, Luke, and Nick Lally. 2021. "For geographical imagination systems." Annals of the American Association of Geographers 111 (1): 26-35. doi:10.1080/24694452.2020.1750941.

Brown, Michael, and Larry Knopp. 2008. "Queering the Map: The Productive Tensions of Colliding Epistemologies." Annals of the Association of American Geographers 98 (1): 40-58. doi: $\underline{10.1080 / 00045600701734042 .}$.

Drucker, Johanna. 2009. SpecLab: Digital Aesthetics and Projects in Speculative Computing. Chicago: University of Chicago Press.

Elwood, Sarah. 2006. "Negotiating Knowledge Production: The Everyday Inclusions, Exclusions, and Contradictions of Participatory GIS Research." The Professional Geographer 58 (2): 197208.

Elwood, Sarah, and Rina Ghose. 2001. "PPGIS in Community Development Planning: Framing the Organizational Context." Cartographica: The International Journal for Geographic Information and Geovisualization 38 (3-4): 19-33. doi:10.3138/R411-50G8-1777-2120.

Gahegan, Mark. 2018. “Our GIS Is Too Small: Our GIS Is Too Small.” The Canadian Geographer / Le Géographe Canadien 62 (1): 15-26. https://doi.org/10.1111/cag.12434.

Gieseking, Jen Jack. 2018. “Operating Anew: Queering GIS with Good Enough Software: Operating Anew.” The Canadian Geographer / Le Géographe Canadien 62 (1): 55-66. doi: $10.1111 /$ cag. 12397.

Guariglia, Matthew. 2020. “Technology Can’t Predict Crime, It Can Only Weaponize Proximity to Policing." Electronic Frontier Foundation. https:/ /www.eff.org/deeplinks/2020/09/technologycant-predict-crime-it-can-only-weaponize-proximity-policing.

Holmes, Brian. 2009. Escape the Overcode: Activist Art in the Control Society. Eindhoven: Van Abbemuseum.

Jefferson, Brian Jordan. 2017a. "Predictable Policing: Predictive Crime Mapping and Geographies of Policing and Race." Annals of the American Association of Geographers, May, 1-16. doi:10.1080/24694452.2017.1293500.

. 2017b. "Digitize and Punish: Computerized Crime Mapping and Racialized Carceral Power in Chicago." Environment and Planning D: Society and Space 35 (5): 775-96. doi: $10.1177 / 0263775817697703$. 
Kitchin, Rob, Justin Gleeson, and Martin Dodge. 2013. "Unfolding Mapping Practices: A New Epistemology for Cartography." Transactions of the Institute of British Geographers 38 (3): 480-96. doi:10.1111/j.1475-5661.2012.00540.x.

Lally, Nick, and Luke Bergmann. 2021. "enfolding: An Experimental geographical imagination system (gis)." Edited by Paul Kingsbury and Anna J. Secor. A Place More Void. University of Nebraska Press.

Lum, Kristian, and William Isaac. 2016. “To Predict and Serve?” Significance 13 (5): 14-19. doi:10.1111/j.1740-9713.2016.00960.x.

McKittrick, Katherine. 2011. “On Plantations, Prisons, and a Black Sense of Place.” Social \& Cultural Geography 12 (8): 947-63. doi:10.1080/14649365.2011.624280.

McPherson, Tara. 2018. Feminist in a Software Lab: Difference + Design. MetaLABprojects. Cambridge, Massachusetts; London, England: Harvard University Press.

Norheim, Robert A. 2001. "How Institutional Cultures Affect Results: Comparing Two OldGrowth Forest Mapping Projects." Cartographica: The International Journal for Geographic Information and Geovisualization 38 (3-4): 35-52. doi:10.3138/4618-2K34-3752-4616.

Poore, Barbara S. 2003. "The Open Black Box: The Role of the End-User in GIS Integration." The Canadian Geographer/Le Géographe Canadien 47 (1): 62-74. doi:10.1111/1541-0064.02e13.

Robinson, Jonnell A., Daniel Block, and Amanda Rees. 2017. "Community Geography: Addressing Barriers in Public Participation GIS." The Cartographic Journal 54 (1): 5-13. doi:10.1080/00087041.2016.1244322.

Rundstrom, Robert A. 1995. "GIS, Indigenous Peoples, and Epistemological Diversity.” Cartography and Geographic Information Systems 22 (1): 45-57. doi:10.1559/152304095782540564.

Schuurman, Nadine. 2000. "Trouble in the Heartland: GIS and Its Critics in the 1990s." Progress in Human Geography 24 (4): 569-90. doi:10.1191/030913200100189111.

Secor, Anna, and Jess Linz. 2017. "Becoming Minor." Environment and Planning D: Society and Space 35 (4): 568-73. doi: $10.1177 / 0263775817710075$.

Sieber, Renee. 2004. "Rewiring for a GIS/2." Cartographica: The International Journal for Geographic Information and Geovisualization 39 (1): 25-39. doi:10.3138/T6U8-171M-452W-516R.

Suchman, Lucy, Jeanette Blomberg, Julian E. Orr, and Randall Trigg. 1999. "Reconstructing Technologies as Social Practice.” American Behavioral Scientist 43 (3): 392-408. doi:10.1177/00027649921955335.

Warren, Gwendolyn. 1971. "About the Work in Detroit.” Field Notes No.3: The Geography of the Children of Detroit. Detroit. 